\title{
1 Phosphorylation at Ser-181 of oncogenic KRAS is required for tumor growth
}

2 Carles Barceló ${ }^{a^{*}}$, Noelia Paco ${ }^{\mathrm{a}^{*}}$, Mireia Morell ${ }^{\mathrm{b}}$, Blanca Alvarez-Moya ${ }^{\mathrm{a}, \mathrm{d}}$, Neus Bota-

3 Rabassedas $^{\mathrm{a}, \mathrm{e}}$, Montserrat Jaumot ${ }^{\mathrm{a}}$, Felip Vilardell ${ }^{\mathrm{c}}$, Gabriel Capella ${ }^{\mathrm{b}}$ and Neus Agell ${ }^{\mathrm{a}, 1}$

4 a Departament de Biologia Cel•lular, Immunologia i Neurociències, Institut

5 d'Investigacions Biomèdiques August Pi i Sunyer (IDIBAPS), Facultat de Medicina.

6 Universitat de Barcelona, C/ Casanova 143, 08036 Barcelona, Spain

7 bereditary Cancer Program, Translational Research Laboratory, Catalan Institute of

8 Oncology, ICO-IDIBELL, Hospitalet de Llobregat, Barcelona, Spain; Gran Via 199-

9 203, 08907- L'Hospitalet de Llobregat Spain

$10{ }^{\mathrm{c}}$ Servei d'Anatomia Patològica i Institut de Recerca Biomèdica de Lleida, Avda. Rovira

11 Roure, 80, 25198 Lleida.

$12{ }^{\mathrm{d}}$ Present address: IFOM-IEO Campus, Milan, Italy

$13 \quad{ }^{\mathrm{e}}$ Present address: IBE, CSIC-UPF, Barcelona, Spain

$14 *$ Both authors contributed equally to this work

15 Corresponding author: Neus Agell, Dept. Biologia Cel·lular, Immunologia i

16 Neurociències, Facultat de Medicina, Universitat de Barcelona, C/Casanova 143,

1708036 Barcelona, Spain; Phone\# 34934036257;e-mail: neusagell@ub.edu.

18 Running title: KRAS phosphorylation promotes tumor growth

19 Keywords: KRAS, tumorogenesis, phosphorylation, PKC

20 Grant support: This study was supported by MICINN-Spain [SAF2010-20712]

21 Conflict of interest: none

22 Word count : 5150

23 Number of figures: 7 


\section{Abstract}

27 KRAS phosphorylation has been reported recently to modulate the activity of mutant

28 KRAS protein in vitro. In this study, we defined S181 as a specific phosphorylation site

29 required to license the oncogenic function of mutant KRAS in vivo. The phosphomutant

30 S181A failed to induce tumors in mice, whereas the phosphomimetic mutant S181D

31 exhibited an enhanced tumor formation capacity, compared to the wild-type KRAS

32 protein. Reduced growth of tumors composed of cells expressing the non-

33 phosphorylatable KRAS S181A mutant was correlated with increased apoptosis.

34 Conversely, increased growth of tumors composed of cells expressing the

35 phosphomimetic KRAS S181D mutant was correlated with increased activation of AKT

36 and ERK, two major downstream effectors of KRAS. Pharmacological treatment with

37 PKC inhibitors impaired tumor growth associated with reduced levels of

38 phosphorylated KRAS and reduced effector activation. In a panel of human tumor cell

39 lines expressing various KRAS isoforms, we showed that KRAS phosphorylation was

40 essential for survival and tumorigenic activity. Further, we identified phosphorylated

41 KRAS in a panel of primary human pancreatic tumors. Taken together, our findings

42 establish that KRAS requires S181 phosphorylation to manifest its oncogenic properties,

43 implying that its inhibition represents a relevant target to attack KRAS-driven tumors. 


\section{Introduction}

RAS proteins are well-known small GTPases involved in the regulation of key signal transduction pathways. Cycling from the inactive (GDP-bound) to the active (GTPbound) state faithfully responds to extracellular signals due to its tight regulation by GTP-exchange factors (GEFs) and GTPase activating proteins (GAPs). Activating point mutations that render RAS proteins insensitive to the extracellular signals are crucial steps in the development of the vast majority of cancers (1-3). Three different genes code for a total of four different Ras isoforms named HRAS, NRAS, KRAS4A and KRAS4B. RAS mutations, mainly at the $K R A S 4 B$ (herein after referred to as $K R A S$ ) genes, occur in pancreatic (95\%), colon (40\%) and adenocarcinomas of the lung (35\%) $(1,4,5)$. The most prevalent oncogenic mutations in $R A S$ at codons 12,13 and 61 preserve the GTP-bound, active state by inhibiting intrinsic GTPase activity or interfering with the action of GAPs. In the GTP-bound form, RAS is able to interact with different effector proteins and consequently activates signal transduction pathways. Among those, the best characterized are the RAF1/MEK/ERK and the phosphatidylinositol-3-kinase (PI3K)/AKT $(6,7)$.

Since oncogenic mutations of KRAS give rise to an always GTP-bound protein which constitutively activates the effectors, positive or negative physiological regulation of oncogenic KRAS was not initially expected. Several reversible posttranslational modifications of KRAS have been described that could modulate KRAS oncogenic activity (8). Ubiquitination of oncogenic KRAS at lysine-147 in the guanine nucleotide-binding motif increases its binding to the downstream effectors PI3K and RAF1 thus increasing its tumorigenic activity (9). Furthermore, acetylation at lysine104 affects interaction with GEFs and inhibits in vitro transforming activity of oncogenic KRAS (10). KRAS has, adjacent of the farnesylated C-terminal cysteine, a 
stretch of six contiguous lysines in a total of eight lysine residues -known as the polybasic domain- which promotes an electrostatic interaction with the negativelycharged phosphate groups of phospholipids $(11,12)$. Phosphorylation of KRAS at serine-181 within this domain has been described (13). We previously reported a role of KRAS Ser181 phosphorylation for activation of the wild-type KRAS in vitro and to regulate also in vitro oncogenic KRAS activity (14). By using both a genetic and pharmacological approach we demonstrate here that phosphorylation of oncogenic KRAS is required for tumor growth in vivo and that also this modification can be detected in human tumors. Furthermore, pharmacological inhibition of oncogenic KRAS phosphorylation suppresses KRAS oncogenic activity.

\section{Materials and Methods}

\section{Antibodies and reagents}

Primary antibodies used for immunoblotting were as follow: Anti-Actin (clone C4) (\#691001; 1:1000; MP Biomedicals, Santa Ana, CA, USA), Anti-GAPDH (\#MAB374; 1:1000; Chemicon, Billerica, MA, USA); Anti-cleaved caspase-3 (Asp175) (\#9661; 1:1000; Cell Signaling, Danvers, MA, USA); Anti-AKT (\#9272; Cell Signaling); Antiphospho-AKT (Thr308) (\#9275; 1:1000, Cell Signaling), Anti-p44/42 MAPK (ERK 1/2) (\#9102; 1:1000; Cell Signaling); Anti-phospho-p44/42 MAPK (ERK 1/2) (Thr202/Tyr204) (\#9101; 1:1000; Cell Signaling), Anti-cyclin B1 (\#4138; 1:1000; Cell Signaling); Anti-KRAS (clone Ab-1) mouse (\#OP24, 1:400, Calbiochem); Anti-PanRas (clone Ab-3) mouse (\#OP40; 1:400; Calbiochem); Anti-HRAS (clone C20) rabbit (\#Sc-520, Santa Cruz); Anti-NRAS (clone F155) mouse (\#Sc-31, Santa Cruz); AntiGAP120 (sc-63; 1:100; Santa Cruz, Santa Cruz, CA, USA); Anti-PKCס (\#610398; 1:500; BD Transduction Laboratories, San Jose, CA); Anti-phospho-PKC $\delta$ 
95 (Ser643/676) (\#9376; 1:1000; Cell Signaling). For immuhistochemistry we used Anti96 Ki-67 (SP6) (\#NM-9106S; 1:200; NeoMarkers, Kalamazoo, MI, USA). We used

97 DeadEnd Colorimetric TUNEL System (G7132; Promega) for the TUNEL assays.

98 The reagents used for the detection of phospho-KRAS were: Protein Phosphatase $\lambda$

99 (\#539514-20KV; Calbiochem); Phos-tagTM (\#AAL-107, Wako Chemicals GmbH,

100 Neuss, Germany).

101 The inhibitors of PKC used were: Bryostatin-1 (\#BIB0342, Apollo Scientific, Chesire,

102 UK), Edelfosine (1-O-Octadecyl-2-O-methyl-glycero-3-phosphorylcholine) (\#BML-

103 L108, Enzo Life Science, Farmingdale, NY, USA), Bisindolylmaleimide I (BIM)

104 (\#CAS 176504-36, Millipore), Gö6983 (\#G1918, Sigma Aldrich).

105

106 Cell lines

107 NIH3T3, SW480, A549, MPanc-96 and HPAF-II cells obtained from American Tissue 108 and Cell Collection (ATCC) were grown in Dulbecco's Modified Eagle's Medium 109 (DMEM) containing 10\% FCS (Biological Industries), and routinely verified according 110 to the specifications outlined in the ATCC Technical Bulletin. NIH3T3 stable cell lines 111 expressing either HA-KRASG12V, HA-KRASG12V-S181A or HA-KRASG12V-

112 S181D were obtained as previously described (14).

113 DLD-1 knock-out of mutant KRAS allele DLD $1^{\text {KRASwt/- }}$ were obtained from Horizon

114 Discovery Ltd (clone D-WT7; \#HD105-002; http://www.horizondiscovery.com;

115 Cambridge, UK). DLD1 ${ }^{\text {KRASwt/- }}$ cells were generated using the proprietary adeno-

116 associated virus (AAV) gene targeting technology GENESIS ${ }^{\circledR}$. Cells were maintained

117 according to the supplier recommendations in McCoy's modified media containing 10\%

118 FBS (Biological Industries). DLD1 ${ }^{\text {KRASwt/- }}$ stable cell lines expressing either HA-

119 KRASG12V, HA-KRASG12V-S181A or HA-KRASG12V-S181D were obtained from 
$120 \mathrm{DLD} 1^{\mathrm{KRASwt} /}$ after transfecting with the specific HA-KRASV12 plasmids (14) and a

121 puromycine resistance plasmid (pSG5A). After selection with puromycine $(4 \mu \mathrm{g} / \mathrm{mL})$

122 clones or pools were obtained. .

123

124 Tumor generation in mice

125 The day of the injection, one million NIH 3T3 cells stably expressing either HA126 KRASG12V, HA-KRASG12V-S181A or HA-KRASG12V-S181D suspended in 0,1

127 mL PBS buffer were subcutaneously injected into both flanks of Swiss nude mice

$128\left(\right.$ foxn $1^{-/}$). Generated tumors were measured over time and at day 18 after injection,

129 mice were euthanized and tumors were harvested, weighed, measured and processed for

130 analysis (each group $\mathrm{n}=10$ ).

131 For DLD-1 xenografts, one million cells stably expressing either HA-KRASG12V or

132 HA-KRASG12V-S181A suspended in 0,1 mL PBS buffer were subcutaneously injected

133 into both flanks of Swiss nude mice $\left(\right.$ foxn $1^{-/}$) Generated tumors were measured over

134 time and at day 28 after injection, mice were euthanized and tumors were harvested,

135 weighed, measured and processed for analysis (each group $\mathrm{n}=10$ ).

136 For the assays with the PKC inhibitors, one million NIH 3T3 cells stably expressing

137 either HA-KRASG12V or HA-KRASG12V-S181D were subcutaneously injected into

138 both flanks of Swiss nude mice. When tumor reached a designated volume of $\sim 150 \mathrm{~mm}^{3}$,

139 animals were randomized and divided into vehicle (DMSO), Bryostatin-1 or Edelfosine

140 treatment groups. Mice were weighed daily and received an intraperitoneal injection of

141 either $75 \mu \mathrm{g} / \mathrm{Kg}$ Bryostatin-1 in $5 \%(\mathrm{v} / \mathrm{v})$ DMSO, $30 \mathrm{mg} / \mathrm{Kg}$ Edelfosine in $5 \%(\mathrm{v} / \mathrm{v})$

142 DMSO or $5 \%(\mathrm{v} / \mathrm{v})$ DMSO (vehicle) for 5 days. At day 6 after the beginning of the

143 treatment, mice were euthanized and tumors were harvested, weighed and processed for 144 analysis. 
145 All mouse experiments were performed in accordance with protocols approved by the

146 Animal Care and Use Committee of ICO-IDIBELL Hospitalet de Llobregat, Barcelona,

147 Spain.

148

149 Sample Lysis, Gel electrophoresis, immunoblotting,

150 Cultured cells were lysed in Ras extraction buffer (20 mM Tris- $\mathrm{HCl}, \mathrm{pH} 7.5,2 \mathrm{mM}$

151 EDTA, $100 \mathrm{mM} \mathrm{NaCl}, 5 \mathrm{mM} \mathrm{MgCl} 2,1 \%(\mathrm{v} / \mathrm{v})$ Triton X-100, $5 \mathrm{mM} \mathrm{NaF}, 10 \%$ (v/v)

152 glycerol and $0.5 \%(\mathrm{v} / \mathrm{v}) 2$-mercaptoethanol) supplemented with a cocktail of protease

153 and phosphatase inhibitors $\left(0.1 \mathrm{mM} \mathrm{Na}_{3} \mathrm{VO}_{4}, 1 \mathrm{mM}\right.$ phenylmethylsulfonyl fluoride, 10

$154 \mathrm{mM} \beta$-glycerophosphate, $2 \mu \mathrm{g} / \mathrm{ml}$ aprotinin and $10 \mu \mathrm{g} / \mathrm{ml}$ leupeptin)

155 Tumors were lysed using Polytron (Fischer Scientific, Pittsburg, PA, USA) in Ras

156 extraction buffer, and protein resolved using standard SDS-PAGE electrophoresis. After

157 electrotransfer, membranes were incubated using the indicated antibodies and then

158 incubated with peroxidase-coupled secondary antibody. Immunocomplexes were

159 detected by enhanced chemiluminescence reaction ECL western blotting analysis

160 system (Amersham Biosciences, Piscataway, NJ, USA) and imaged by LAS-3000

161 (Fujifilm, Tokyo, Japan). When required, band intensity was determined using the

162 measurement tool of Multigauge 2.0 (FUJIFILM, Tokyo, Japan).

\section{Cell viability assay (MTT)}

165 Human cell lines or DLD-1 expressing the same amounts of HA-KRASG12V and HA-

166 KRASG12V-S181D were seeded at $10^{4}$ cells per p96 with DMEM 10\% FCS. Next day,

167 were treated with the corresponding concentration of PKC inhibitors for $48 \mathrm{~h}$. Then, 10

$168 \mu \mathrm{L}$ of AB solution (MTT Cell Growth Assay Kit, \#CT02, Millipore) were added to each

169 well and incubated at $37^{\circ} \mathrm{C}$ for $4 \mathrm{~h}$. Then, $0.1 \mathrm{~mL}$ isopropanol with $0.04 \mathrm{~N} \mathrm{HCl}$ was 
170 added and mixed thoroughly. Absorbance was measured with a test wavelength of 570

$171 \mathrm{~nm}$ and a reference wavelength of $630 \mathrm{~nm}$ according to manufacturer's

172 recommendations.

173

\section{Measurement of Ras isoform activation}

175 RBD (Ras-binding domain of Raf-1) pull-down assays were performed as previously

176 described (14) to determine the amount of active K-, H- and NRAS.

178 Histology

179 Mice tumors were embedded either in paraffin or frozen in OCT. Paraffin sections were

180 stained following the haematoxylin-eosin standard protocol to study their histological

181 appearance. Mitotic count in 5 consecutive high-power fields (100x) was performed to

182 compare the mitotic index between groups. Frozen section in OCT were used to

183 determine apoptosis by TUNEL assay following manufacturers recommendations

184 (Roche), and to determine the percentage of proliferating cells by immunohistochemistry using Ki-67 antibodies.

\section{Human tumors}

188 Five biopsies of Human Pancreatic Ductal Adenorcarcinoma obtained by 189 Doudenopancreatectomy were orthotopically implanted to nude mice and were 190 perpetuated at least four passages. All patients gave informed written consent to 191 participate and to have their biological specimens analyzed. The study was cleared by 192 the Ethical Committee of Hospital de Bellvitge. 
195 Phos-tag ${ }^{\mathrm{TM}}$ SDS-PAGE. To detect phospho-KRAS from human tumor samples and

196 from nude mice grafts, a fragment of $\sim 0,1 \mathrm{~g}$ from a tumor biopsy was homogenized in $1970,4 \mathrm{~mL}$ of $\lambda$ Phosphatase Lysis Buffer (50 mM Tris- $\mathrm{HCl} \mathrm{pH} 8 ; 150 \mathrm{mM} \mathrm{NaCl}, 2 \mathrm{mM}$ 198 EDTA, 10\% Glycerol, 1\% Nonidet P40, $5 \mathrm{mM}$ DTT, 2mM $\mathrm{MnCl}_{2}$ ) containing either 199 protease inhibitor cocktail (Halt Protease Inhibitor Cocktail, \#87786, Thermo Scientific, 200 Rockford, IL USA) alone or plus phosphatase inhibitors (0.2 $\left.\mathrm{mM} \mathrm{Na}_{3} \mathrm{VO}_{4}, 5 \mathrm{mM} \mathrm{NaF}\right)$.

201 For human cell lines, a $10 \mathrm{~cm}$ dish was homogenized in the $\lambda$ Phosphatase Lysis Buffer 202 as described above. Then, samples homogenized with only protease inhibitors were 203 treated with recombinant Protein Phosphatase $\lambda$ for 30 minutes at $30^{\circ} \mathrm{C}$ according to 204 manufacturer instructions, and finally all tubes were balanced with phosphatase 205 inhibitors in order to equalize both lysis buffers.

206 Protein content was assessed by Lowry method (Lowry et al, 1951) and tubes were 207 balanced. $10 \mu \mathrm{g}$ protein were loaded into a 12\%-polyacrilamide SDS-PAGE gel 208 supplemented with $100 \mu \mathrm{M}$ Phos-tag ${ }^{\mathrm{TM}}$ and $100 \mu \mathrm{M} \mathrm{MnCl}_{2}$ (according to Phos-tag $^{\mathrm{TM}}$ 209 SDS-PAGE protocol indicated by manufacters). The gel was run over night at $5 \mathrm{~mA} / \mathrm{gel}$ 210 and soaked in a general transfer buffer containing $1 \mathrm{mM}$ EDTA for 20 min followed by

21110 minutes incubation with a transfer buffer without EDTA. Then, gels were transferred 212 over night at $50 \mathrm{~V}$ into a PDVF membrane that was blocked and blotted with anti213 KRAS (\#OP24, Calbiochem).

214 Two-dimensional gel electrophoresis (2-DE). $100 \mu \mathrm{g}$ of tumor extract prepared as 215 indicated above, were diluted to a final concentration of $7 \mathrm{M}$ urea, $2 \mathrm{M}$ thiourea, $4 \%$ 216 CHAPS \{3-[(3-cholamidopropyl)-dimethylammonio]-1-propanesulfonate $\}, 65 \mathrm{mM}$

217 DTE, 0.1\% ampholytes (Bio-Lyte 3/10, no. 163-1113; Bio-Rad) and 1.2\% DestreakTM 218 Reagent (GE Healthcare, 17-6003-18) to $125 \mu \mathrm{L}$ volume. Two-dimensional first219 dimension electrophoresis was performed as isoelectric focusing (IEF) with precast, 
220 immobilized pH gradient (IPG) gel strips (ReadyStripTM IPG Strip, $7 \mathrm{~cm}, \mathrm{pH} 7$ to 10;

221 no. 163-2005 [Bio-Rad]) by using a PROTEAN IEF system (Bio-Rad). Sample

222 application and rehydration of the strips were carried out using the active method (50 V

223 contant) according to the manufacturer's instructions (Bio-Rad). Next focusing was

224 performed at 8000 to $20000 \mathrm{~V}$ per hour. IEF gels were equilibrated for $10 \mathrm{~min}$ in a

225 buffer containing $6 \mathrm{M}$ urea, $0.375 \mathrm{M}$ Tris [pH 8.8], 2\% SDS, 20\% glycerol, and 2\%

$226[\mathrm{wt} / \mathrm{vol}] \mathrm{DTE}$, and the second-dimension run was carried out in SDS-polyacrylamide

227 gels. After electrophoresis, gels were transferred to PDVF membranes (Millipore) and

228 immunoblotted with antibodies against KRAS

229

230 Statistics

231 All analyses were performed with GraphPad Prism 5.0. Data represent mean \pm SEM.

232 Mann-Whitney test was used to analyze significance levels. Specific significance levels 233 are found in figure legends. $P<0.05$ was considered significant. 
236 Results and Discussion

237 Oncogenic KRAS phosphorylation at Ser-181 is required for tumor growth

238 To test the prediction that phosphorylation at Ser181 of oncogenic KRAS was required

239 to support tumor growth, NIH3T3 stable cell lines expressing similar levels of 240 oncogenic HA-tagged G12V KRAS, namely HA-KRAS-G12V-S181 (S181), non-

241 phosphorylatable HA-KRAS-G12V-S181A (S181A) or phosphomimetic HA-KRAS-

242 G12V-S181D (S181D) (Fig. 1A) were subcutaneously injected into nude mice and 243 tumor growth was monitored over time. Tumor formation was nearly abolished in cells 244 expressing non-phosphorylatable S181A (Fig. 1B,C and tables S1 and S2). Furthermore, 245 a dramatic increase in tumor growth was observed for phosphomimetic S181D mutant 246 compared to the phosphorylatable S181. No tumor growth was observed when injecting 247 NIH3T3 cells stably expressing wild-type HA-KRAS (Table S1), which confirmed that 248 both engraftment and growth was driven by our oncogenic KRAS phosphomutants. 249 Interestingly, in spite of the dramatic diminished growth of non phosphorylatable 250 S181A derived tumors, KRAS oncoprotein was overexpressed in those tumors 251 compared to the S181 or S181D derived tumors (Fig. 1D). This suggests that, during the 252 process of tumor development, cells with higher expression of non-phosphorylatable 253 KRAS are positively selected in an attempt to overcome the lower tumorogenic activity 254 exhibited by this mutant. Similar results were obtained when injecting in nude mice 255 two independently immortalized S181A clones with distinct expression levels. Again in 256 S181A clones, tumor growth was highly compromised irrespectively of the KRAS 257 protein expression level (Fig. S1).

258 The impaired tumor growth of the non-phosphorylatable S181 G12V mutant associated 259 with a distinct histological pattern. S181A tumors were composed mostly by cells with 
260 an epithelioid appearance and with a significant lymphocytic infiltration (S181A 10.00

$261 \pm 1.08$ lymphocytes per x100 field vs S181 $2.25 \pm 0.63$ vs S181D $1.25 \pm 0.25$,

262 p $<0.0001$ ) (Fig. 3 and Fig.S3). Of note, the same histology has been previously reported

263 for sarcomas harboring the mild KRAS codon 13 mutations (15). In contrast, S181 and

264 S181D derived sarcomas were composed of a fusocellular population showing a

265 hemangiopericitoid patterns. The non-phosphorylatable S181A tumors had also a lower

266 mitotic rate $(\mathrm{S} 181 \mathrm{~A} 4.00 \pm 1.53$ mitotic cells per x100 field vs $27.00 \pm 4.12$ for $\mathrm{S} 181 \mathrm{vs}$

$26754.75 \pm 4.99$ for S181D, $<<0.0001)$ and were the only tumors showing detectable

268 levels of cleaved-caspase 3, a bona fide apoptosis marker (Fig. 1D and Fig S2A),

269 together with a significant increase in TUNEL positive cells (Fig. 2A, B.). This is in

270 accordance with the decreased resistance to apoptosis already reported in vitro for

271 S181A compared to S181 and S181D oncogenic KRAS mutants (14). In agreement with

272 the prediction of a stronger activity of KRAS upon phosphorylation, the

273 phosphomimetic S181D derived tumors exhibited higher ERK and AKT activity (Fig.

274 1D), accompanied by a pronounced increase in the number of positive cells for the

275 proliferative marker Ki-67 (Fig. 2A,B). Although mild increase in TUNEL positive

276 cells was also observed in S181D compared to S181 tumors, S181A tumors were the

277 ones exhibiting the highest degree of apoptosis. Intriguingly, S181A tumors showed

278 higher cyclin B1 expression than the others (Fig. 1D) in line with the requirement of

279 increased Cyclin B1 for apoptosis induction previously reported in several tumor cell

280 lines (16-18). Moreover, Cyclin B1 overexpression has already been related to the mild

281 transforming phenotype of codon 13 KRAS mutations in NIH3T3 models (15).

282 Thus, the impossibility of phosphorylating oncogenic KRAS dramatically changes

283 growth pattern rendering activating mutations much less aggressive and demonstrating

284 the relevance of this posttranslational modification in KRAS-driven transformation. 
286 PKC inhibitors diminish oncogenic KRAS-mediated tumor growth

287 The dependence of oncogenic KRAS on S181 phosphorylation makes oncogenic KRAS

288 a putative target for protein kinase inhibitors. Since PKCs are considered to be the

289 putative kinases for KRAS Ser181 phosphorylation $(13,19,20)$ we tested whether

290 treatment with two general PKC inhibitors that are clinically relevant (Bryostatin-1 and

291 Edelfosine) (21-25) were able to revert tumor growth in a dephosphorylation-dependent

292 manner.

293 Bryostatin-1 inhibits PKC activity when administrated in vitro at concentrations as low 294 as $0.1 \mathrm{nM}(21)$. In our experiments we used $75 \mu \mathrm{g} / \mathrm{Kg}$, a dose that was previously used 295 for in vivo PKC inhibition (26). As shown in Fig. 4B, Bryostatin-1 treatment 296 significantly reduced tumor growth of S181 whereas no effect was evident on "non297 dephosphorylatable" S181D tumors. Of note, we found that Bryostatin-1 treatment, in 298 accordance to its general PKC inhibitor activity, efficiently downregulated both total 299 and active PKC $\delta$ levels as previously described (21) (Fig. 4C). Tumor reduction with 300 Bryostatin-1 treatment was associated with a decreased ERK activity that was specific 301 for S181 phosphorylatable mutant. Moreover, apoptosis was induced as shown by an 302 increase of cleaved caspase-3 levels and TUNEL positive cells (Fig. 4C,D and Fig. 303 S2B). Concomitantly, cell proliferation was inhibited (Fig. 4D.) while cyclin B1 304 expression was increased (Fig. 4C). In this way, Bryostatin-1 treatment showed high 305 specificity for the dephosphorylatable S181 tumors and interestingly, treatment of these 306 tumors efficiently recapitulated the growth and signaling pattern of S181A tumors

307 (21)shown in Fig.1. Concordantly, PKC inhibition did not affect growing and signaling 308 pattern, nor increased apoptosis in the non-dephosphorylatable KRAS S181D tumors. 
309 To further confirm the striking results obtained with Bryostatin-1 treatment on mice we

310 treated the same stable transfected NIH3T3 mice grafts with Edelfosine. This is an ether

311 lipid analog to HMG with reported strong PKC inhibitor activity both in vitro $(27,28)$

312 and in vivo (29). As shown in Fig S4, we reproduced a significantly reduced tumor

313 growth of S181 and again no significant effect was observed in "non-

314 dephosphorylatable" S181D tumors.

315 Altogether, these results suggested that both Bryostatin-1 and Edelfosine, by blocking

316 PCK activity, impair tumor growth inducing KRAS dephosphorylation and subsequent

317 apoptosis. To formally prove this hypothesis, detection of KRAS phosphorylation was

318 necessary. Since no suitable antibodies are available, we used the Phos-Tag ${ }^{\mathrm{TM}}$-based

319 approach $(30,31)$ to determine the oncogenic KRAS phosphorylation status in the

320 generated tumors. This method is based on the fact that a complex formation between

321 the phosphate group of a phosphorylated protein and a divalent metal ion in Phos-TagTM

322 reduces the mobility of the phospho-protein during the electrophoresis separation, thus

323 allowing resolution of phosphorylated and non-phosphorylated proteins into different

324 bands. As shown in Fig. 4E, a slow migrating band of HA-KRAS could be observed in

325 the tumors generated from cells expressing the S181 oncogenic KRAS, that was absent

326 in S181D tumors. Disappearance of this band upon $\lambda$ Phosphatase treatment

327 corroborated it was phosphorylated KRAS. Most interestingly, in Bryostatin-1 and

328 Edelfosine treated animals phosphorylated KRAS was no longer observed (Fig.4E and

329 Fig.S4). Together, these observations reinforce the notion that PKC-dependent Ser181-

330 phosphorylation of oncogenic KRAS is required for tumorigenesis. This effect may

331 account for the previously reported inhibition of different KRAS-driven tumor

332 xenografts by PKC pharmacologic inhibition $(29,32,33)$. Interestingly, it has also been 
333 shown that PKC $\delta$ knock-down prevents apoptosis and promotes tumorigenesis in cells

334 addicted to aberrant KRAS signaling (34-36).

335

336 Human cell lines require phosphorylation of KRAS for survival and tumor growth

337 In order to determine whether the requirement for KRAS phosphorylation observed in

338 our NIH3T3 KRAS-transformation model was also involved in human cell lines

339 tumorigenesis, we ectopically expressed the HA-KRAS-G12V phosphomutants

340 described above in the human colorectal cancer cell line DLD-1 but previously

341 knocked-out for the oncogenic endogenous KRAS allele (DLD1 ${ }^{\mathrm{KRASwt} /}$ ).

342 We found that under serum-saturating growth conditions $(10 \% \mathrm{FCS})$, human colon

343 cancer cells DLD-1 expressing S181A mutant exhibited a significantly reduced growth

344 compared to phosphomimetic S181D expressing cells (Fig. 5A). Trying to recapitulate

345 tumor growth conditions, we evaluated cell growth under serum-limiting conditions

346 (0.1\% FCS). After 4 days of starvation, cells stably expressing S181A showed

347 significantly higher reduced growth under serum starvation culture conditions compared

348 to S181 and phosphomimetic S181D (Fig. 5A,). Moreover, S181A exhibited increased

349 sensitivity to apoptosis under serum deprivation or by adriamycin- induced genotoxic

350 damage (Fig 5A,B), thus demonstrating a pro-apoptotic effect of the S181A oncogenic

351 KRAS.

352 In order to evaluate real tumorigenic capacity of these cells, subcutaneous injection of

353 DLD1 ${ }^{\text {KRASwt/- }}$ expressing either HA-KRASG12V or HA-KRASG12V-S181A

354 phosphomutants was performed. S181A derived tumors were significantly smaller than

355 S181 tumors (Fig. 5C). This confirmed the requirement of KRAS S181 phosphorylation

356 for tumorigenesis of human colon cell lines. 
357 A preferential activation of endogenous wild-type H- and N-RAS alleles induced by the 358 oncogenic KRAS has recently been reported (37). To check whether diminished growth

359 capacity of S181A was due to lack of activation of the endogenous RAS isoforms,

360 RBD pull-down assays were performed to test GTP loading of endogenous RAS

361 isoforms. Lower GTP loading of endogenous Ras in the S181A expressing cells was not

362 observed compared with the other phosphomutants (Fig. S5).

363 Next, we wanted to determine whether BIM and Gö6983, two PKC inhibitors $(38,39)$

364 were able to affect DLD-1 cells in a KRAS S181-specific manner. To this aim, dose-

365 response to these PKC inhibitors was evaluated in DLD $1^{\text {KRASwt/- }}$ cells expressing HA-

366 KRASG12V and using DLD-1 expressing HA-KRASG12V-S181D as a non-

367 dephosphorylatable control. After $48 \mathrm{~h}$ of treatment, it was shown that at doses between

$3681 \mu \mathrm{M}$ and $20 \mu \mathrm{M}$ for BIM and $1.5 \mu \mathrm{M}$ and $10 \mu \mathrm{M}$ for Gö6983, cells expressing oncogenic

369 KRAS with S181 exhibited significantly enhanced sensitivity to PKC inhibition

370 compared to the phosphomimetic non-dephosphorylatable mutant (Fig 6A). Most

371 importantly, after PKC inhibition, S181 cells lost its KRAS phosphorylation as shown

372 by Phos-tag ${ }^{\text {TM }}$ SDS-PAGE gels (Fig 6A).

373 Finally, we evaluated the ability of a set of PKC inhibitors to reduce proliferation

374 together with KRAS phosphorylation in a panel of human cell lines from different

375 origin harboring oncogenic KRAS. We found that at doses reported to inhibit PKC (21,

$37628,38,40)$, cell growth was compromised. Most importantly, after $12 \mathrm{~h}$ of treatment,

377 band corresponding to phospho-KRAS was lost, thus reinforcing the idea that PKC

378 inhibition is able to revert growth in a KRAS S181-dependent manner (Fig. 6B).

379

380 KRAS is phosphorylated in human tumors. 
381 Next, we investigated whether the S181 phosphorylation observed in our model system

382 was also present in human tumors. To do so, a set of orthotopic xenografts derived from 383 carcinomas of the exocrine pancreas were analyzed. Five tumors harboring codon 12

384 KRAS mutations were tested. As shown in Fig. 7, by using Phos-Tag ${ }^{\mathrm{TM}}$ SDS-PAGE,

385 several bands were detected in all tumors using the anti-KRAS antibody. Treatment

386 with $\lambda$ phosphatase (Fig. 7) and two-dimensional electrophoresis analysis corroborated

387 the presence of phosphorylated KRAS in these human tumors (Fig. S6). Thus, the

388 presence of phospho-KRAS in human malignancies emphasizes the alleged requirement

389 of this modification for human KRAS-driven tumorigenesis.

390 Altogether, the results depict a scenario of a novel tight regulation of KRAS

391 oncogenicity by phosphorylation at S181. We have recently shown that although

392 phosphorylated KRAS is mainly found at the plasma membrane $(40,41)$,

393 phosphorylated K-Ras forms distinct plasma membrane signaling platforms that induce

394 preferential activation of main KRAS effectors involved in oncogenesis . Interestingly,

395 this distinct functionality could be reverted by PKC inhibitors (40). This would give a

396 rationale for the strikingly different tumorigenic activity of oncogenic KRAS according

397 to its S181 phosphorylation status .

398 The fact that, as we show, this could be efficiently pharmacologically inhibited raises

399 the possibility of novel therapeutic strategies targeting KRAS-driven human

400 malignancies. So far, clinical trials with PKC regulators have been disappointing

401 mostly because of the lack of selectivity and unacceptable toxicity (39). The

402 identification of KRAS as a key PKC target may help in developing specific inhibitors

403 of KRAS phosphorylation. 
Author Manuscript Published OnlineFirst on December 26, 2013; DOI: 10.1158/0008-5472.CAN-13-1750

Author manuscripts have been peer reviewed and accepted for publication but have not yet been edited.

406 Grant Support

407 This study was supported by MICINN-Spain [SAF2010-20712]. Carles Barceló and

408 Noelia Paco are recipient of pre-doctoral fellowships from MEC-Spain, and the Catalan

409 Government respectively.

410 Research. 
413 1. Bos JL. Ras oncogenes in human cancer: A review. Cancer Res 1989;49:4682-9.

414 2. Malumbres M, Barbacid M. RAS oncogenes: The first 30 years. Nat Rev Cancer $415 \quad 2003 ; 3: 459-65$.

416 3. Downward J. Control of ras activation. Cancer Surv 1996;27:87-100.

417 4. Prior IA, Lewis PD, Mattos C. A comprehensive survey of ras mutations in cancer.

418 Cancer Res 2012;72:2457-67.

419 5. Schubbert S, Shannon K, Bollag G. Hyperactive ras in developmental disorders and 420 cancer. Nat Rev Cancer 2007;7:295-308.

421 6. Shields JM, Pruitt K, McFall A, Shaub A, Der CJ. Understanding ras: 'It ain't over 'til 422 it's over'. Trends Cell Biol 2000;10:147-54.

423 7. Marshall CJ. Ras effectors. Curr Opin Cell Biol 1996;8:197-204.

424 8. Ahearn IM, Haigis K, Bar-Sagi D, Philips MR. Regulating the regulator: Post-

425 translational modification of RAS. Nat Rev Mol Cell Biol 2011;13:39-51.

426 9. Sasaki AT, Carracedo A, Locasale JW, Anastasiou D, Takeuchi K, Kahoud ER, et al. 427 Ubiquitination of K-ras enhances activation and facilitates binding to select downstream 428 effectors. Sci Signal 2011;4:ra13.

429 10. Yang MH, Nickerson S, Kim ET, Liot C, Laurent G, Spang R, et al. Regulation of 430 RAS oncogenicity by acetylation. Proc Natl Acad Sci U S A 2012;109:10843-8. 
431 11. Hancock JF, Magee AI, Childs JE, Marshall CJ. All ras proteins are

432 polyisoprenylated but only some are palmitoylated. Cell 1989;57:1167-77.

433 12. Silvius JR. Mechanisms of ras protein targeting in mammalian cells. J Membr Biol $434 \quad 2002 ; 190: 83-92$.

435 13. Ballester R, Furth ME, Rosen OM. Phorbol ester- and protein kinase C-mediated 436 phosphorylation of the cellular kirsten ras gene product. J Biol Chem 1987;262:268843795.

438 14. Alvarez-Moya B, Lopez-Alcala C, Drosten M, Bachs O, Agell N. K-Ras4B

439 phosphorylation at Ser181 is inhibited by calmodulin and modulates K-ras activity and 440 function. Oncogene 2010;29:5911-22.

441 15. Guerrero S, Figueras A, Casanova I, Farre L, Lloveras B, Capella G, et al. Codon 12 442 and codon 13 mutations at the K-ras gene induce different soft tissue sarcoma types in 443 nude mice. FASEB J 2002;16:1642-4.

444 16. Yuan J, Yan R, Kramer A, Eckerdt F, Roller M, Kaufmann M, et al. Cyclin B1 445 depletion inhibits proliferation and induces apoptosis in human tumor cells. Oncogene $446 \quad 2004 ; 23: 5843-52$.

447 17. Chu R, Terrano DT, Chambers TC. Cdk1/cyclin B plays a key role in mitotic arrest448 induced apoptosis by phosphorylation of mcl-1, promoting its degradation and freeing 449 bak from sequestration. Biochem Pharmacol 2012;83:199-206.

450 18. Borgne A, Versteege I, Mahe M, Studeny A, Leonce S, Naime I, et al. Analysis of 451 cyclin B1 and CDK activity during apoptosis induced by camptothecin treatment.

452 Oncogene 2006;25:7361-72. 
19. Bivona TG, Quatela SE, Bodemann BO, Ahearn IM, Soskis MJ, Mor A, et al. PKC regulates a farnesyl-electrostatic switch on K-ras that promotes its association with bcl-

455 XL on mitochondria and induces apoptosis. Mol Cell 2006;21:481-93. 103.

463 Antitumor alkyl-lysophospholipid analog edelfosine induces apoptosis in pancreatic

464 cancer by targeting endoplasmic reticulum. Oncogene 2012;31:2627-39. reversal effect on cancer multidrug resistance. Mar Drugs 2012;10:2312-21.

468 Bryostatin analogue-induced apoptosis in mantle cell lymphoma cell lines. Exp Hematol $469 \quad 2012 ; 40: 646,56 . \mathrm{e} 2$. cytotoxicity of anti-CD22 immunotoxin is enhanced by bryostatin 1 in B-cell

472 lymphomas through CD22 upregulation and PKC-betaII depletion. Haematologica $473 \quad 2012 ; 97: 771-9$. 
26. Mohammad RM, Li Y, Mohamed AN, Pettit GR, Adsay V, Vaitkevicius VK, et al.

475 Clonal preservation of human pancreatic cell line derived from primary pancreatic

476 adenocarcinoma. Pancreas 1999;19:353-61. comparative study of the effect of the antineoplastic ether lipid 1-O-octadecyl-2-Omethyl-glycero-3-phosphocholine and some homologous compounds on PKC alpha and

480 PKC epsilon. Biochim Biophys Acta 2005;1687:110-9.

28. Zhou X, Arthur G. 1-O-octadecyl-2-O-methylglycerophosphocholine inhibits protein kinase C-dependent phosphorylation of endogenous proteins in MCF-7 cells. Biochem J 1997;324:897-902. induced by protein kinase $\mathrm{C}$ inhibition and application to human tumor cell lines. J Cell Physiol 2004;198:277-94. phosphoproteins using phos-tag SDS-PAGE. Nat Protoc 2009;4:1513-21.

490 Phosphorylation of claudin-2 on serine 208 promotes membrane retention and reduces

491 trafficking to lysosomes. J Cell Sci 2012;125:4902-12. 
33. Jasinski P, Zwolak P, Terai K, Borja-Cacho D, Dudek AZ. PKC-alpha inhibitor

497 MT477 slows tumor growth with minimal toxicity in in vivo model of non-ras-mutated

498 cancer via induction of apoptosis. Invest New Drugs 2011;29:33-40.

35. Shen L, Kim SH, Chen CY. Sensitization of human pancreatic cancer cells harboring mutated K-ras to apoptosis. PLoS One 2012;7:e40435.

504 Protein kinase C delta is a downstream effector of oncogenic K-ras in lung tumors.

505 Cancer Res 2011;71:2087-97. oncogenic ras is essential for tumorigenesis. Nat Commun 2012;3:1168.

509 involves two distinct mechanisms and promotes breast cancer cell survival. Biochim

510 Biophys Acta 2013;1830:4040-5.

511 39. Mochly-Rosen D, Das K, Grimes KV. Protein kinase C, an elusive therapeutic

512 target? Nat Rev Drug Discov 2012;11:937-57.

513 40. Barcelo C, Paco N, Beckett AJ, Alvarez-Moya B, Garrido E, Gelabert M, et al.

514 Oncogenic K-ras segregates at spatially distinct plasma membrane signaling platforms

515 according to its phosphorylation status. J Cell Sci 2013;126:4553-9. 
516 41. Lopez-Alcala C, Alvarez-Moya B, Villalonga P, Calvo M, Bachs O, Agell N.

517 Identification of essential interacting elements in K-ras/calmodulin binding and its role

518 in K-ras localization. J Biol Chem 2008;283:10621-31.

519

520 Research. 
522 Fig. 1. Phosphorylation at Ser181 is necessary for tumor growth of oncogenic

523 KRAS-G12V. NIH 3T3 cells stably expressing either, HA-KRAS-G12V (S181), HA-

524 KRAS-G12V-S181A (S181A) or HA-KRAS-G12V-S181D (S181D) were injected into

525 each flank of nude mice (each group $n=10$ ). A) Levels of exogenous HA-KRAS from

526 the different NIH 3 T3 pools were analyzed by immunoblot the day of injection into

527 mice. Pool S181\#2 was injected into mice (herein after referred to as S181); B) Tumor

528 volumes were measured at days 13, 16 and 19 after injection; C) At day 19 mice were

529 euthanized and tumors were dissected and weighed. Graph showing the weight of

530 excised tumors (each dot corresponds to a tumor); D) Total cell lysates of representative

531 excised tumors were immunoblotted to detect the indicated proteins (numbers indicate

532 different tumors). Anti-GAP120 was used as loading control;

533 Fig. 2. Lack of growth of non-phospohorylatable KRAS tumors correlates with 534 decreased proliferation (Ki-67) and increased apoptosis (TUNEL) markers. A)

535 Tumor sections were stained for Ki-67 or TUNEL. Scale bars represent $50 \mu \mathrm{m} ; \mathrm{B})$

536 Quantifications of Ki-67 labeling (left) and TUNEL labeling (right) were made from at

537 least 2 different tumors per mutant (each point represents a counted field). (***,

$538 \mathrm{p}<0.0001, * *, \mathrm{p}<0.001$ and $*, \mathrm{p}<0.01, \mathrm{p}$ for Student's two-tailed t test; mean and SEM

539 are represented).

540 Fig. 3. Differential tumor growth according to KRAS phosphorylation is associated

541 with a distinct histological patern. Paraffin sections were stained following the

542 haematoxylin-eosin protocol to study their histological appearance. Arrow caps indicate

543 lymphocyte infiltration. Scale bars represent $50 \mu \mathrm{m}$.

544 Fig. 4. Pharmacologic inhibition of PKC activity inhibits tumor growth and 545 KRAS-G12V dependent signaling pathways in a K-RasG12V Ser181546 phosphorylation dependent manner. NIH 3T3 cells stably expressing either HA547 KRAS-G12V (S181); or HA-KRAS-G12V-S181D (S181D) were injected into each 548 flank of nude mice. When tumor reached a designated volume of $\sim 150 \mathrm{~mm}^{3}$ (latency 549 time shorter for S181D tumors), animals were divided into two groups (each group $550 \mathrm{n}=10)$ and treated daily either with vehicle (5\% DMSO) or Bryostatin-1 (Bryo) (75 $551 \mu \mathrm{g} / \mathrm{Kg}$ ) for 6 days, and euthanized next day. A) Western blot showing HA-K-Ras 
552

553

554

555

556

557

558

559

560

561

562

563

564

565

566

567

568

569

570

571

572

573

574

575

576

577

578

579

580

581

582

expression in different pools of NIH 3T3 cells the day of injection. Injected NIH 3T3 pools (red arrows) were chosen among the ones with equivalent expression for HAKRAS-G12V (S181) or HA-KRAS-G12V-S181D (S181D); B) Increment in tumor size was obtained by comparing tumor volume at the starting day (day 1) and at day 7 of treatment. Dissected tumors from the nude mice are displayed below the graph. Scale bar represents $25 \mathrm{~mm} \mathrm{C)} \mathrm{Total} \mathrm{cell} \mathrm{lysates} \mathrm{of} \mathrm{representative} \mathrm{excised} \mathrm{tumors} \mathrm{were}$ immunoblotted to detect the indicated proteins (numbers indicate different tumors). Anti-GAP120 was used as loading control D) Quantifications of Ki-67 labeling (left) and TUNEL labeling (right) were made from at least 2 different tumors per mutant (each point represents a counted field). (***, p<0.0001, **, p $<0.001$ and $*, p<0.01, \mathrm{p}$ value for Student's two-tailed t test; ns: non-significant differences; mean and SEM are represented). E) Cellular extract from tumors were resolved in Phos-Tag ${ }^{\mathrm{TM}}$ SDS-PAGE gels and immunoblot was performed using anti-HA antibody. An aliquot of a S181 tumor from an animal treated with DMSO was incubated, prior to electrophoresis, at $30^{\circ} \mathrm{C}$ with phosphatase $\lambda(\lambda)$ or only with buffer $(\mathrm{Ctl})$ to discard unspecific effects due to heating samples.

Fig. 5. Phosphorylation at Ser181 is necessary for cell proliferation, survival and tumor growth of DLD1 KRAS wt/- cell expressing KRASG12V phosphomutants. A) $3 \cdot 10^{4}$ DLD1 KRAS wt/- cells stably expressing either HA-KRASG12V-S181A, HAKRASG12V-S181D or HA-KRASG12V-S181 were cultured under serum-saturated ( $10 \%$ FCS) or serum-limiting $(0.1 \%$ FCS $)$ conditions for 4 days and counted to evaluate the proliferation rate. KRAS expression of the different mutants at the initial day and levels of cleaved caspase $3(\mathrm{Cl}$. casp 3$)$ at the fourth day are showed. B) The different mutants were cultured for 2 days with adryamicin $(5 \mu \mathrm{M})$ to induce genotoxic damage. The sensitivity to apoptosis was analyzed by the levels of cleaved caspase 3 ( $\mathrm{Cl}$. casp 3). C) Pools of DLD1 ${ }^{\text {KRAS wt- }}$ expressing HA-KRAS-G12V S181 or HA-KRAS-G12V S181A were injected into each flank of nude mice. Levels of exogenous HA-KRAS from the pools injected were analyzed by immunoblot the day of injection into mice. At day 28 after injection mice were euthanized and tumors were weighed. Graph showing the weight of excised tumors. 
583 Fig.6. Pharmacologic PKC inhibition impairs KRAS phosphorylation and cell

584 survival. A) DLD1 ${ }^{\text {KRAS wt/- }}$ expressing KRASG12V -S181 or KRASG12V-S181D (as

585 non-dephosphorylatable control) were treated with the PKC inhibitors Gö6938 (Gö)

$586(1.5,5$ and $10 \mu \mathrm{M})$ and BIM $(1,10$ and $20 \mu \mathrm{M})$ for 48 hours. The columns represent

587 the growth rate estimated by the measurement of the absorbance following the MTT

588 assay as a function of the initial cell number (left). Extracts from DLD1 KRAS wt/-

589 expressing KRASG12V -S181 or KRASG12V-S181D treated or no with phosphatase $\lambda$

$590(\lambda)$ or with PKC inhibitors (Gö6983 or BIM) were resolved in Phos-Tag ${ }^{\text {TM }}$ SDS-PAGE

591 gel following by immunoblotting using anti-KRAS antibodies (right).B) A pannel of

592 human cell lines from different origin harboring oncogenic KRAS were treated with 5

$593 \mu \mathrm{M}$ BIM, $1 \mu \mathrm{M}$ Gö6983 (Gö), $1 \mu \mathrm{M}$ Bryostatin-1(Bryo) and $10 \mu \mathrm{g} / \mathrm{mL}$ Edelfosine

594 (Edelf) for 48h. Cells were harvested and extracts were resolved in Phos-Tag ${ }^{\text {TM }}$ SDS-

595 PAGE and immunoblotted using anti-KRAS antibody.

596 Fig. 7. Detection of the phosphorylated form of oncogenic KRAS in human

597 pancreatic ductal adenocarcinomas. Extract from 5 different human pancreatic ductal

598 adenocarcinomas with oncogenic mutations in codon 12 of KRAS (\#1 G12D

599 heterozygous; \#2 G12D heterozygous; \#3 G12D homozygous; \#4 G12V heterozygous;

600 \#5 G12V heterozygous), were resolved in Phos-Tag ${ }^{\mathrm{TM}}$ SDS-PAGE or SDS-PAGE

601 followed by immunoblotting using anti-KRAS antibodies. An aliquot of each extract

602 was previously incubated with phosphatase $\lambda$. Anti-GAP120 was used as loading

603 control. 

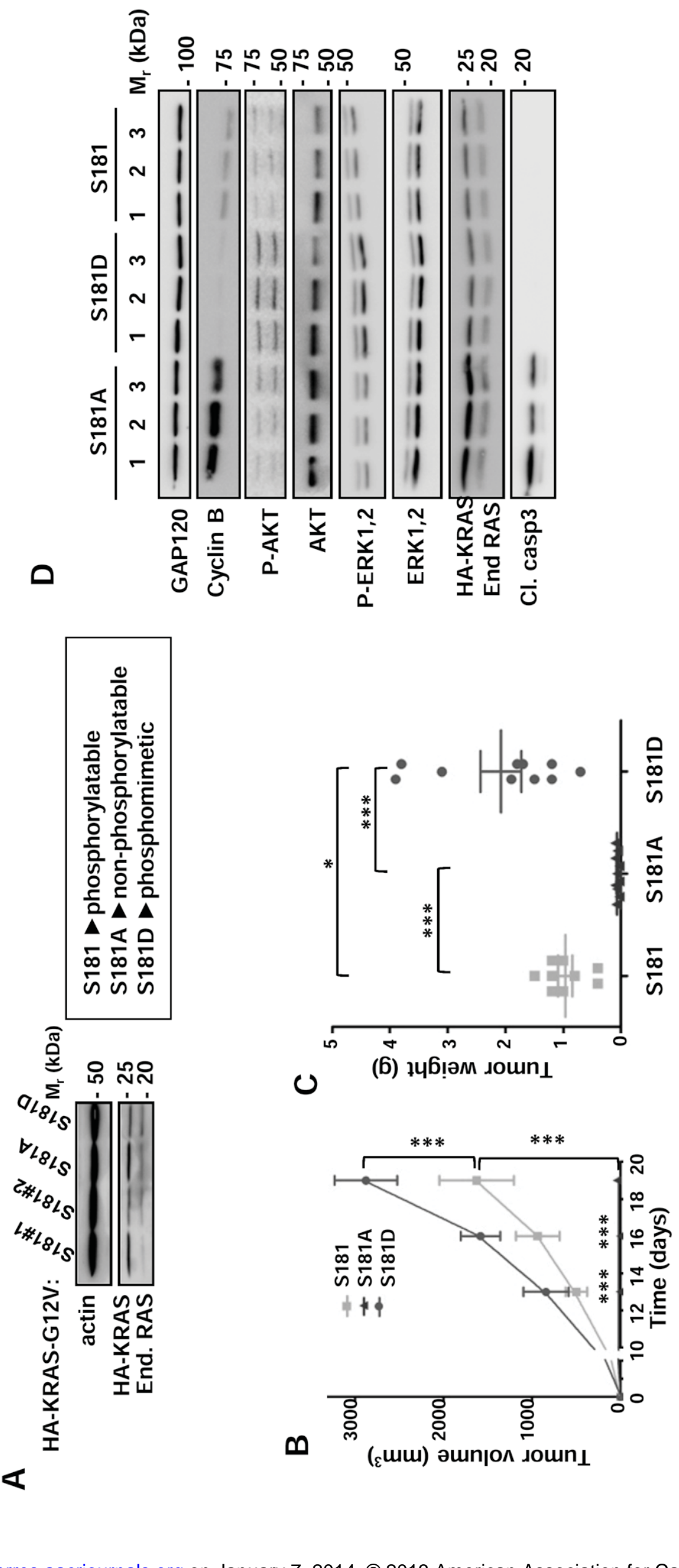

Downtoaded from cancerres.aacrjournals.org on January 7, 2014. (C) 2013 American Association for Cancer Research. 


\section{Fig 2}

A

Ki67
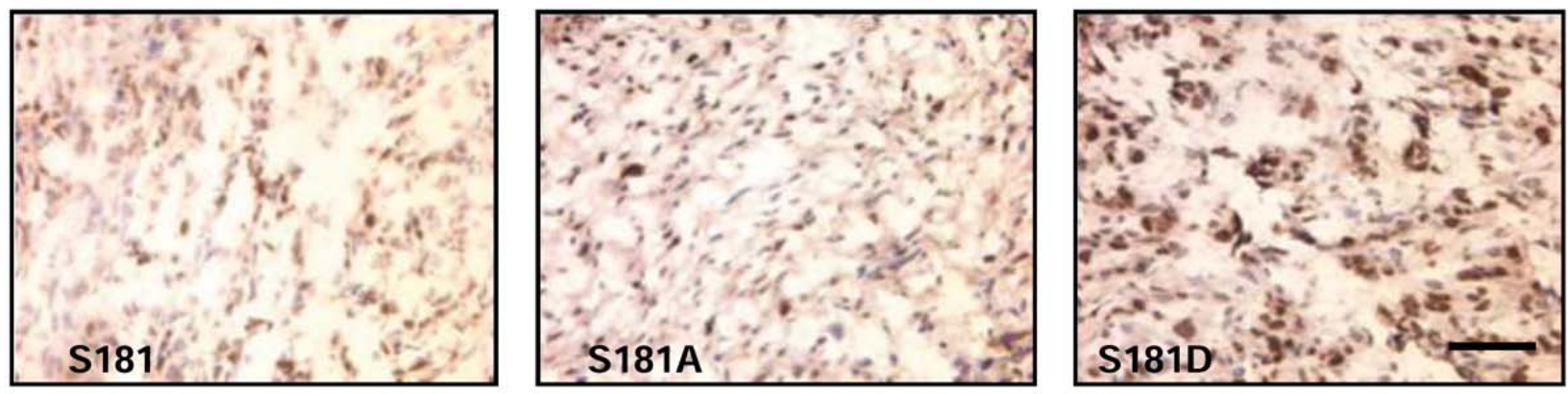

\section{TUNEL}
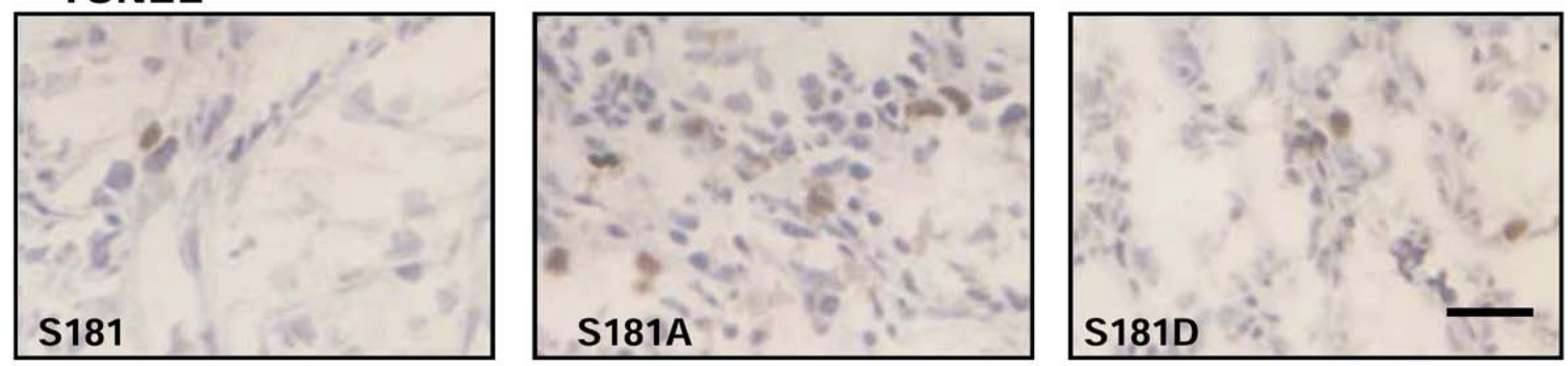

B
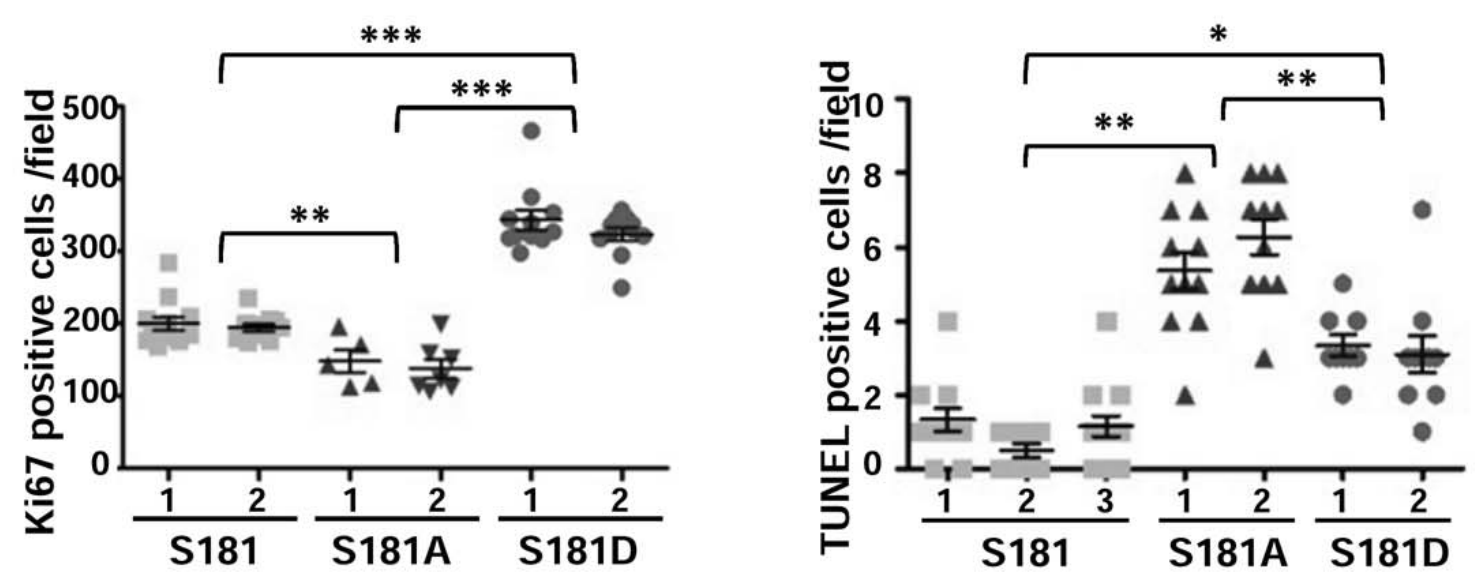
Author Manuscript Published OnlineFirst on December 26, 2013; DOI: 10.1158/0008-5472.CAN-13-1750

Author manuscripts have been peer reviewed and accepted for publication but have not yet been edited.

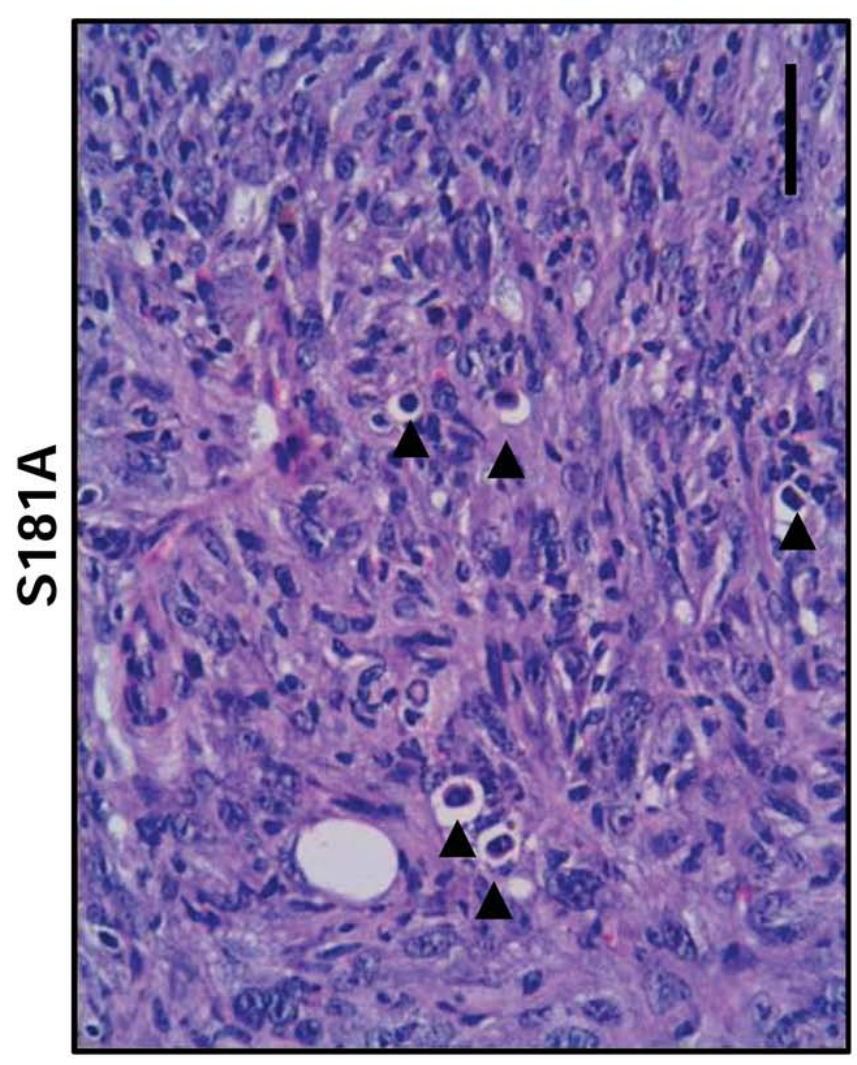

$m$
$\frac{20}{L}$
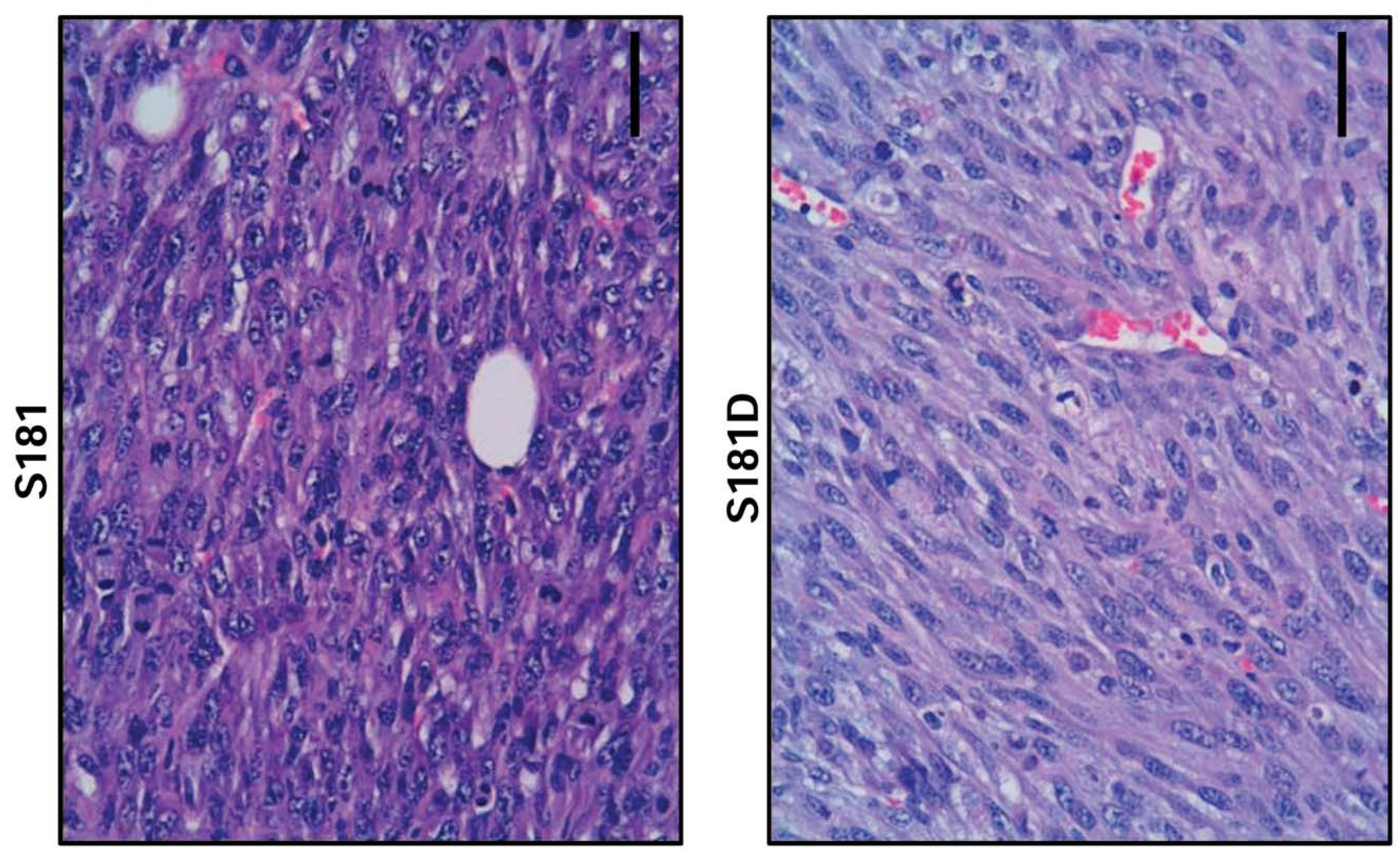


\section{Fig 4}

A

C

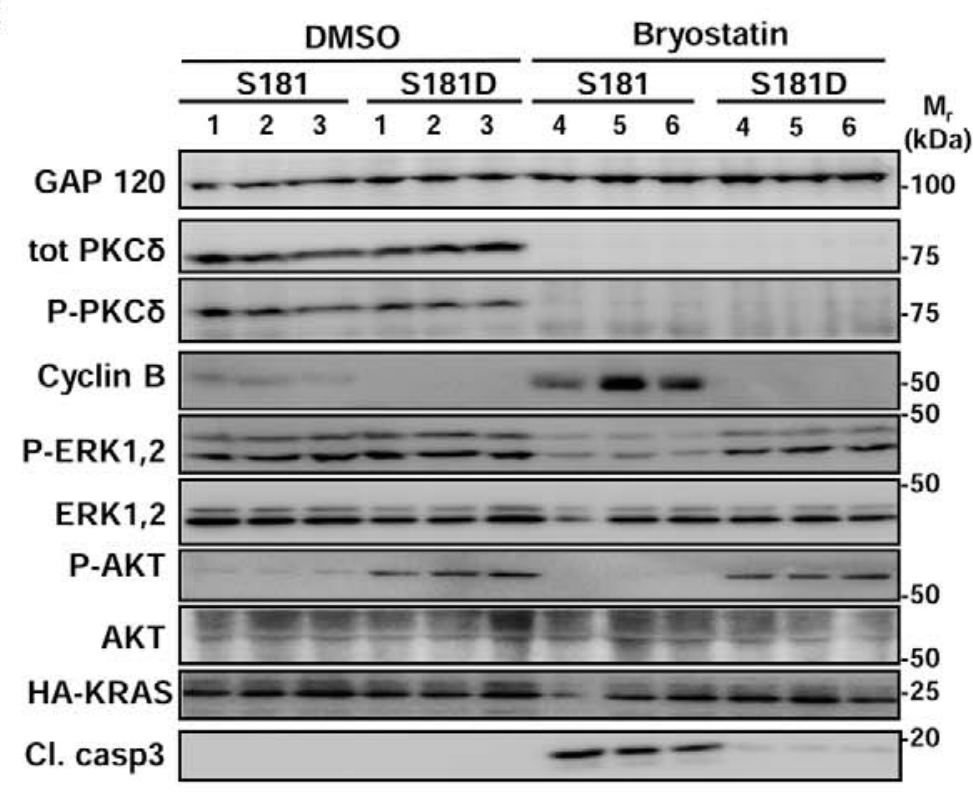

E

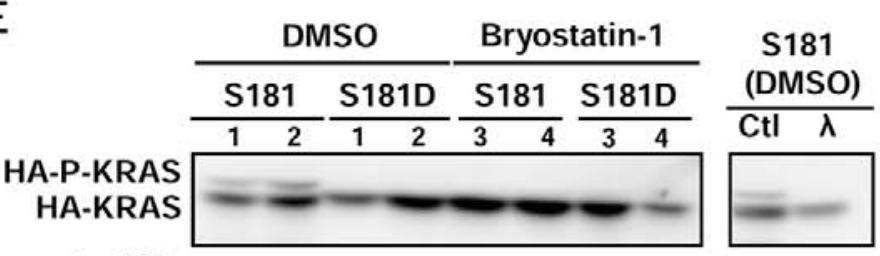

Anti-HA
B
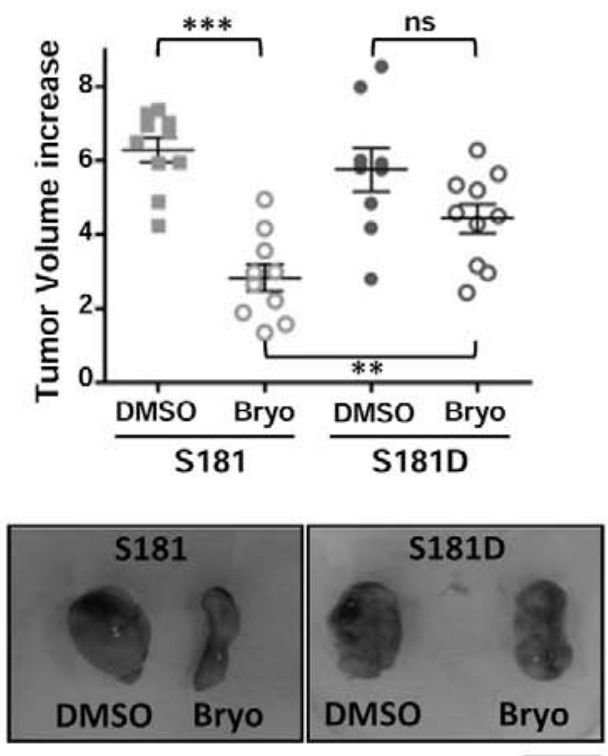

D
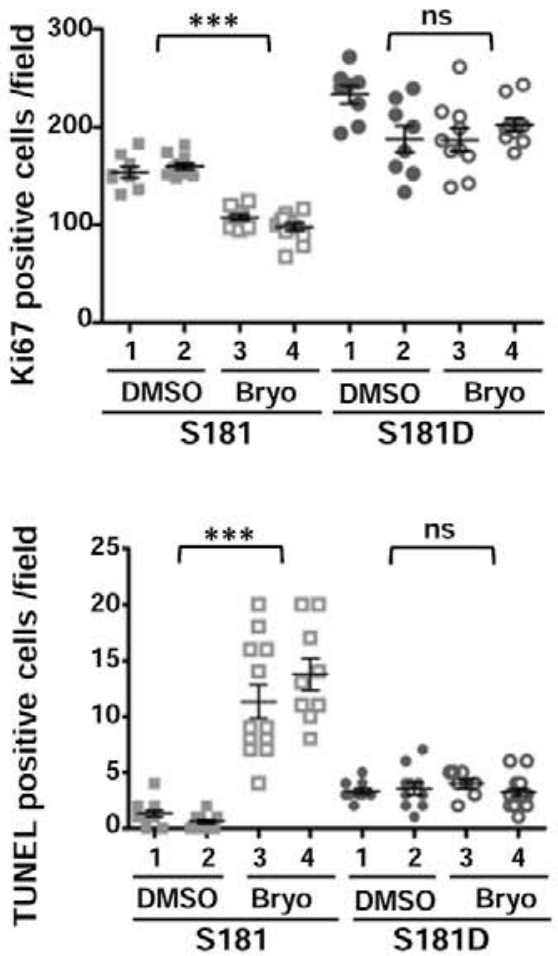

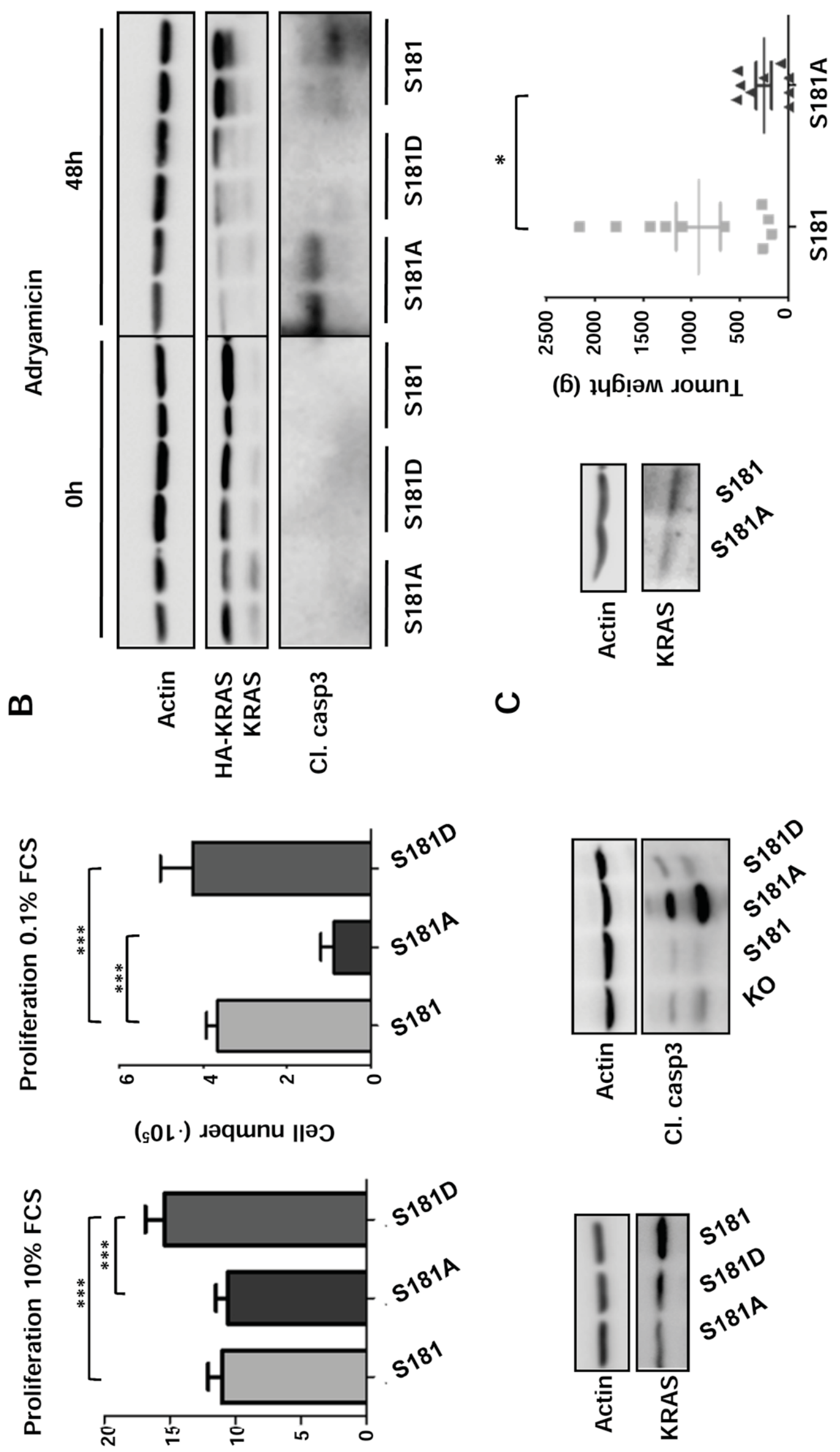

(s0L.) มəqunu ॥əכ

$\tau$ Research. 

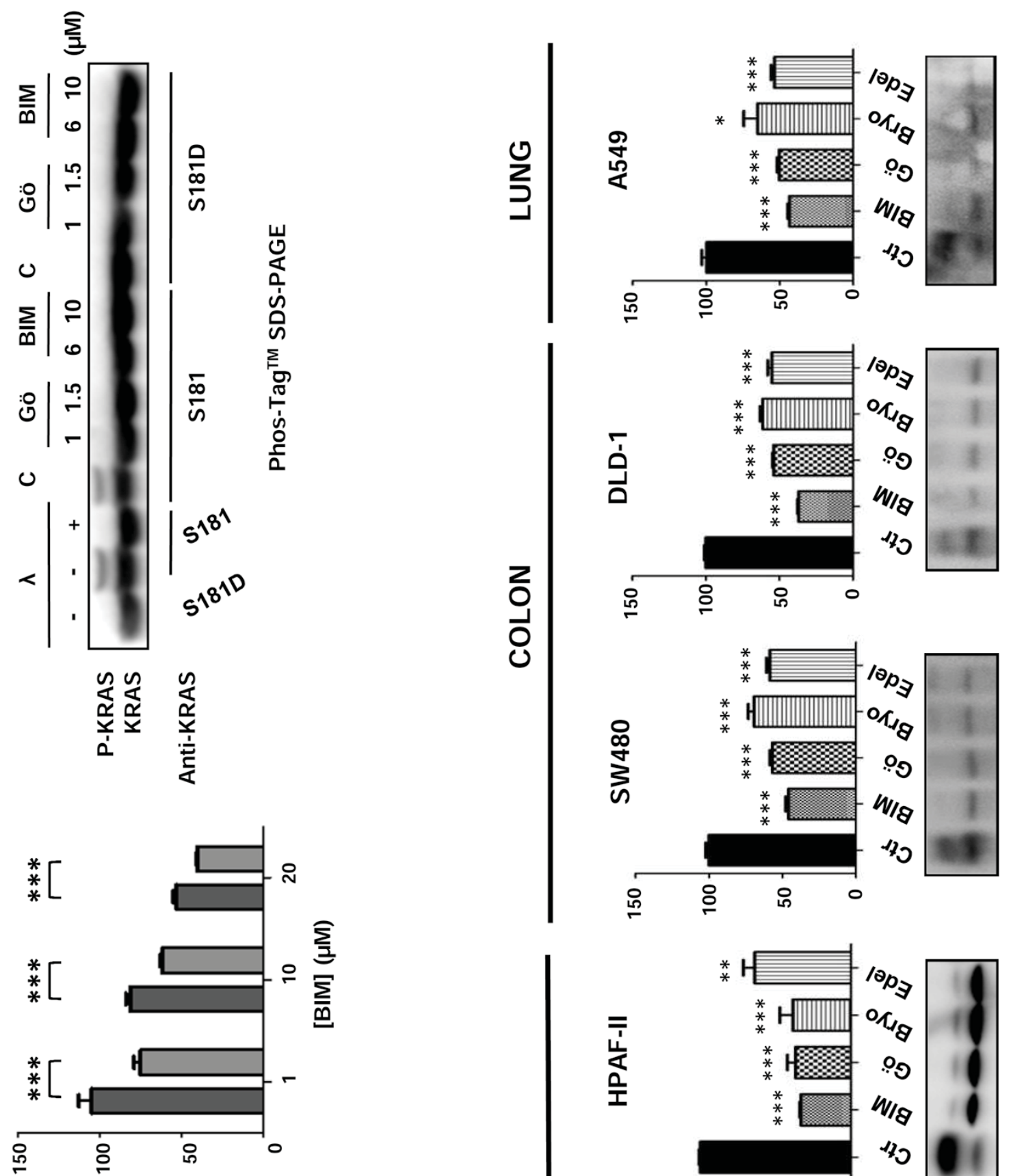

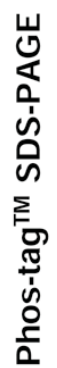

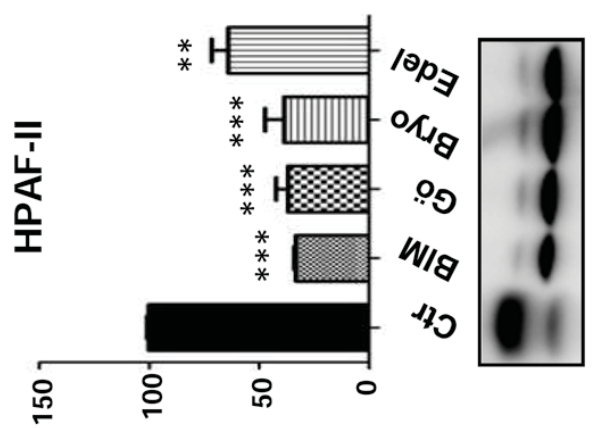

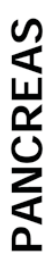

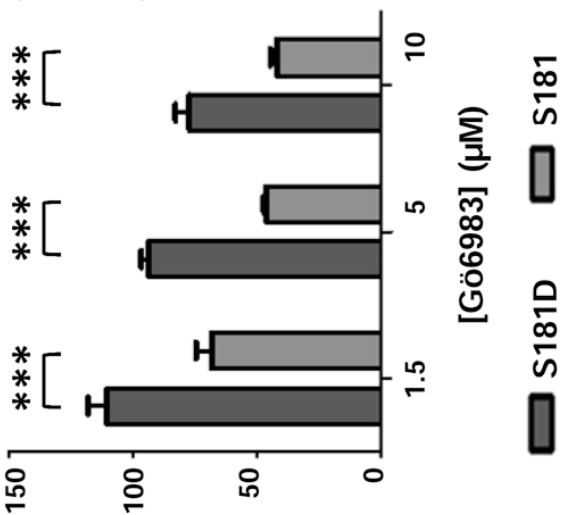

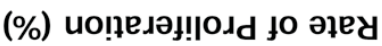

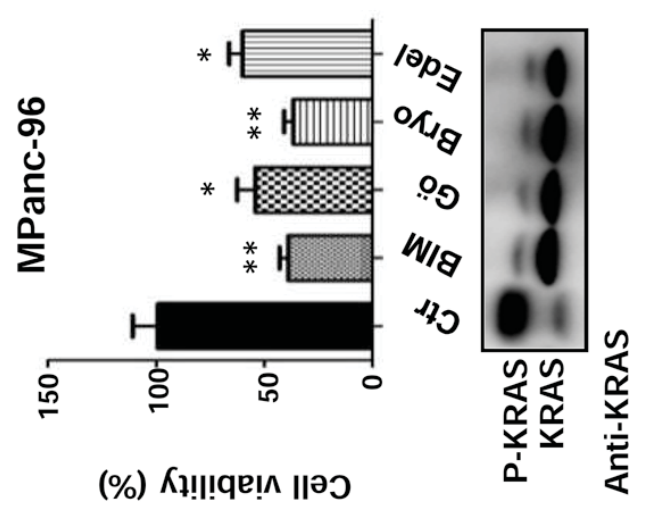




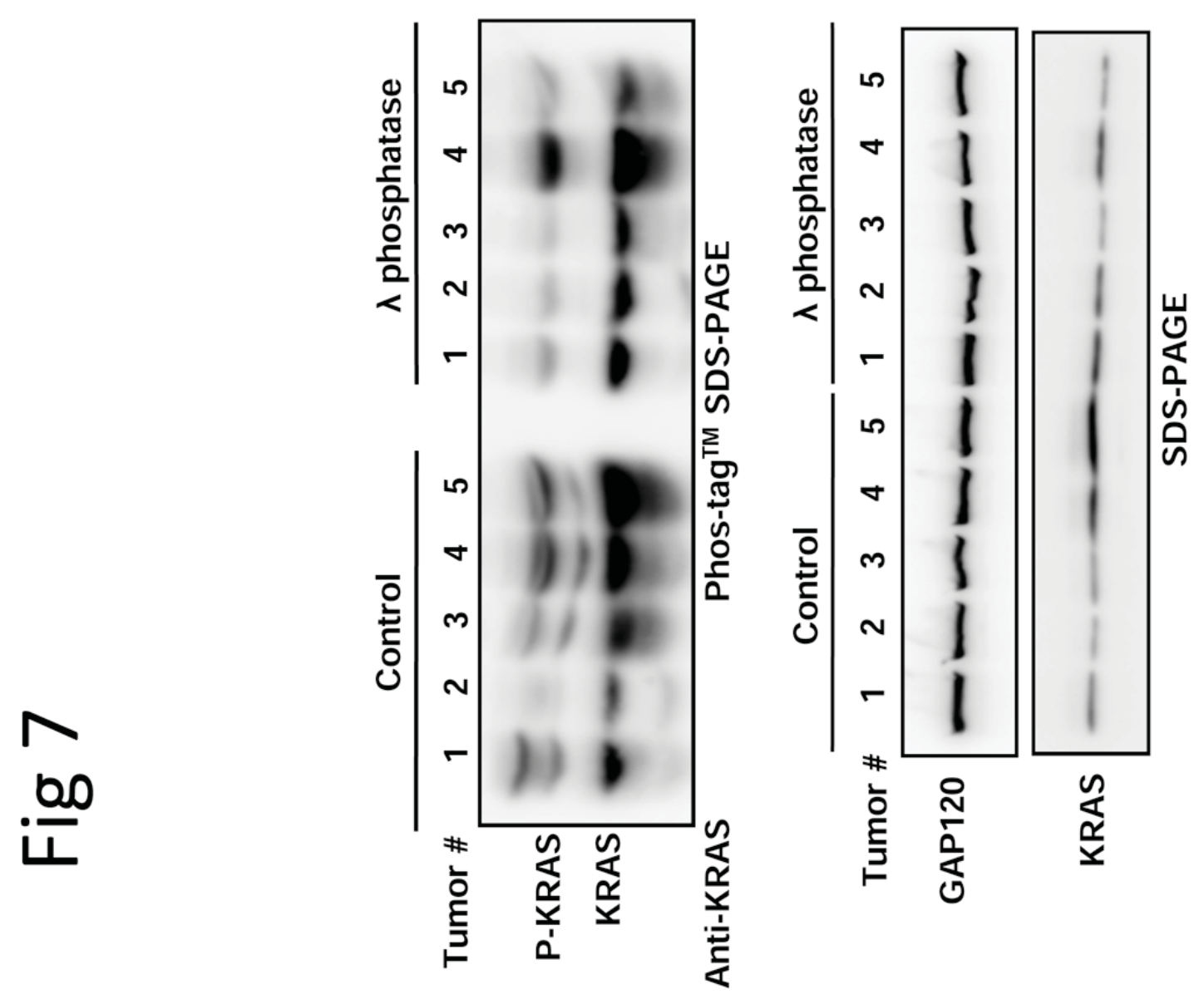
Research. 